\title{
Estudo do movimento de um duplo cone sobre uma pista em $\mathrm{V}$ (Study of the motion of a double cone on a $V$ track)
}

\author{
F. Pascoal ${ }^{\mathbb{W}}$, S.J. Prado, E.A. y Castro \\ Faculdade de Ciências Integadas do Pontal, Universidade Federal de Uberlândia, Ituiutaba, MG, Brasil \\ Recebido em 27/1/14; Aceito em 18/4/14; Publicado em 31/7/2014
}

\begin{abstract}
Utilizamos o princípio da conservação de energia mecânica para estudar o movimento de um duplo cone sobre uma pista em V para diferentes condições (condições iniciais e configurações geométricas). Mostramos que, dependendo das configurações geométricas, esse problema apresenta três regimes de movimentos. No mais interessante deles, o duplo cone rola para a parte mais elevada da pista em $\mathrm{V}$, até mesmo se dermos a ele um pequeno impulso na direção oposta. Por fim, apresentamos o aparato experimental que utilizamos para verificar alguns de nossos resultados teóricos.
\end{abstract}

Palavras-chave: duplo cone, cone de Leybourn, conservação de energia mecânica.

We use the law of conservation of mechanical energy in order to study the motion of a double cone on a $\mathrm{V}$ track under different conditions (initial conditions and geometric configuration). We show that, depending on the geometric configurations, this problem exhibits three regimes of motion. At the most interesting one, the paradoxical regime, the double cone rolls to the upper section of the $\mathrm{V}$ track, even when we give it a small impulse in the opposite direction. In addition, we present the experimental system that we used to verify some of our theoretical results.

Keywords: double cone, Leybourn uphill roller, conservation of mechanical energy.

\section{Introdução}

O experimento do duplo cone é um dos mais intrigantes experimentos demonstrativos de mecânica. Um corpo rígido similar a dois cones idênticos unidos pela base, o duplo cone, é posto em uma rampa em V, e, se as configurações geométricas do sistema forem propícias, ao soltarmos o duplo cone, ele rola para a parte mais elevada da rampa. Como veremos mais a frente nesse artigo, este aparente desafio a lei da gravidade tem uma explicação: apesar do duplo cone estar subindo a rampa, seu centro de massa está na verdade descendo.

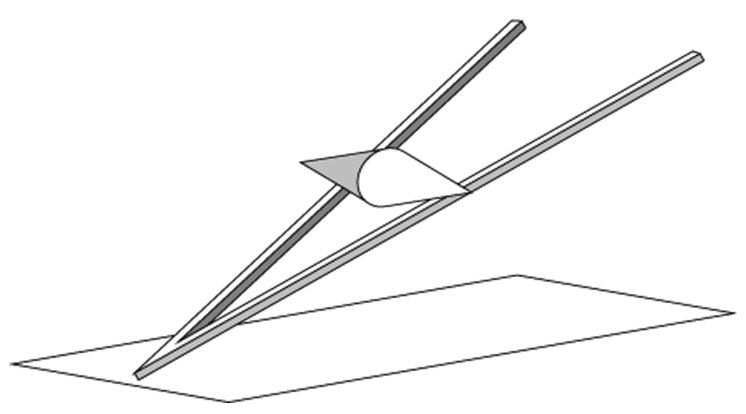

Figura 1 - Vista em perspectiva da rampa e do duplo cone em contato.

\footnotetext{
${ }^{1}$ E-mail: fabiopr@pontal.ufu.br.

A história da origem do experimento do duplo cone e sua popularização é abordada na Ref. [四]. Seus autores apontam que, embora não haja nenhum documento que confirme isso, alguns estudiosos creditam a criação do experimento do duplo cone aos membros da Accademia del Cimento entre 1657 a 1667, enquanto outros atribuem a criação à Galileu. Os autores deste mesmo artigo preferem creditar a autoria do experimento do duplo cone ao francês Jean Theophile Desaguliers em 1734 [च]. Existe um registro escrito sobre o experimento do duplo cone mais antigo que o de Desaguliers, no entanto, a autoria desse trabalho é um tanto misteriosa. na Ref. [3], o autor identificado apenas como J.P. apresenta um artigo de duas páginas entitulado $A M e$ chanical Paradox or a New and Diverting Experiment Whereby a Heavy Body fhall by its own Weight move up a floping Afcent. Nele, J.P. apresenta em detalhes como construir um duplo cone e dois trilhos inclinados sobre os quais ele rolaria para a parte mais elevada. J.P. ainda apresenta a explicação física baseada na "queda" do centro de massa para explicar o fenômeno. Curiosamente, não é difícil encontrar quem se refira a este experimento como Leybourn's uphill roller, embora William Leybourn, em pricípio, não tenha escrito esta parte do livro. 
Muito também já foi explorado no que se refere a criação do experimento do duplo cone a partir de materiais de baixo custo. Na Ref. [G], os autores apresentam uma forma alternativa para confecciona-lo a partir de tábuas de madeira e funis de plástico. Nas Refs. [5] e [6] as listas de materiais são ainda mais simples: na primeira referência, utilizam-se 2 garrafas pet com a parte superior cônica, 2 varetas de madeira ou arame rígido e fita crepe; na segunda, apenas papel, cola e tesoura.

As condições geométricas para que o paradoxo gravitacional ocorra são estudadas nas Refs. [ய, [, [], no entanto, existem algumas discordâncias nos resultados. Mais a frente, retomaremos esta discussão e apontaremos qual deles está correto, bem como o erro cometido pelos demais.

Conhecemos dois artigo que dizem respeito ao movimento do duplo cone. Na Ref. [8], os autores utilizam a conservação de energia para obter numericamente as evoluções temporais do ponto de contato no duplo cone, da velocidade do centro de massa e da velocidade angular do mesmo. No artigo citado, os cálculos são feitos para uma configuração geométrica e uma condição inicial muito específicas: A configuração geométrica é correspondente ao aparato experimental que eles dispunham e a condição inicial é que o duplo cone é solto do repouso do começo da rampa. Na Ref. [ర], os autores apresentam uma vasta discussão sobre a dinâmica do problema do duplo cone, no entanto, o artigo fica muito restrito aos cálculos. Poucas soluções numéricas são apresentadas fazendo com que a compreensão dos resultados físicos fique um tanto difícil.

Em nosso trabalho, utilizaremos o princípio da conservação de energia mecânica para estudar o movimento de um duplo cone sobre uma pista em V. Mostraremos que, variando as configurações geométricas, o problema do duplo cone apresenta três regimes de movimento: ordinário, indiferente e paradoxal. Abordaremos cada um desses regimes separadamente. Por fim, apresentaremos o aparato experimental que utilizamos para verificar alguns de nossos resultados teóricos.

\section{Energia mecânica}

Em nossos cálculos, consideraremos que a rampa em $\mathrm{V}$ em questão tem um ângulo de abertura $2 \beta$. O ângulo de ascensão $\alpha$ da mesma e o coeficiente de atrito estático são tais que o duplo cone rola sem deslizar sobre a rampa. No caso de rolamento sem deslizamento, a força de atrito estática não realiza trabalho [0, [0]]. Além disso, estamos desconsiderando quaisquer outras forças dissipativas, portanto, a energia mecânica do duplo cone é conservada.

A energia mecânica do sistema é dada por

$$
E=\frac{m}{2} v_{c m}^{2}+\frac{I}{2} \omega^{2}+V
$$

onde $v_{c m}$ e $\omega$ são, respectivamente, a velocidade do cen- tro de massa e a velocidade angular de rotação do duplo cone. Os dois primeiros termos da Eq. (四) correspondem a energia cinética de translação e a energia cinética de rotação. O terceiro termo é a energia potencial gravitacional.

Para especificar a posição o duplo cone, utilizaremos as variáveis $\mathbf{s}, \mathbf{l}, \mathbf{r}$, o ângulo $\gamma$ e a altura de contato $z$, que estão devidamente representadas na Figs. 2, 3 e 4. O vetores $\mathbf{s}$ e $\mathbf{l}$ são componentes ortogonais do vetor que liga o vértice da pista em $\mathrm{V}$ ao ponto de contato. Os dois vetores pertencem ao plano da pista em $\mathrm{V}$ e o vetor $\mathbf{l}$ é paralelo ao eixo de rotação do duplo cone. O vetor $\mathbf{r}$ liga o ponto de contato ao eixo de rotação do duplo cone. Como veremos mais a frente, a condição de rolar sem deslizar implica um valor não nulo para $\gamma$, o que pode parecer estranho frente a nossas experiências prévias com problemas de plano inclinado.

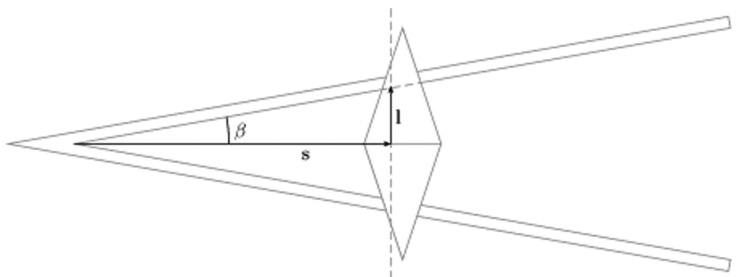

Figura 2 - Vista superior no plano da rampa do duplo cone em contato com a pista em $\mathrm{V}$.

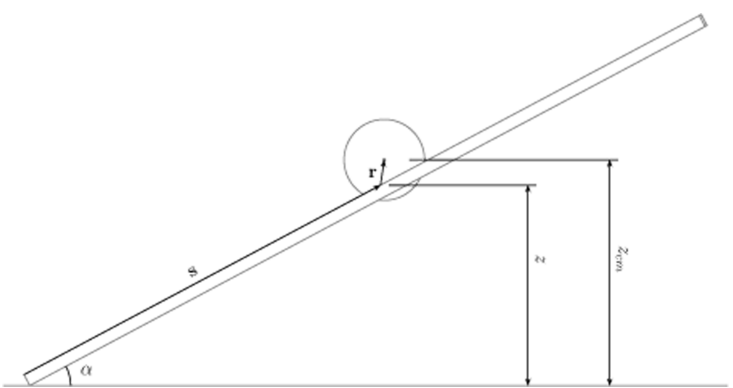

Figura 3 - Vista lateral da rampa e do duplo cone em contato.

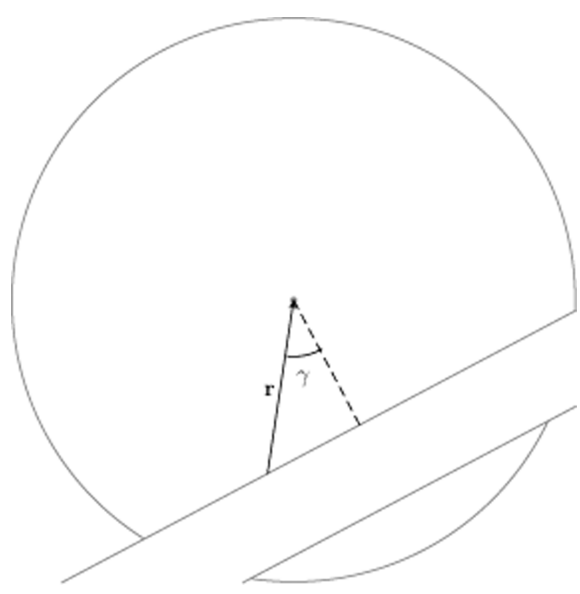

Figura 4 - Detalhe ampliado da vista lateral da rampa e do duplo cone em contato.

Nosso trabalho agora é explorar a geometria e os vínculos do problema para reescrever a expressão 
da energia mecânica (四) em termos de apenas uma variável, a variável $z$. Comecemos por escrever $s, l$ e $r$ em função de $z$. Como podemos ver da Fig. 2,

$$
s=\frac{z}{\operatorname{sen} \alpha} .
$$

Da Fig. 3,

$$
l=s \tan \beta=z \frac{\tan \beta}{\operatorname{sen} \alpha} .
$$

Da geometria do duplo cone (Fig. 5),

$$
r=R\left(1-\frac{l}{L}\right)=R\left(1-\frac{z}{L} \frac{\tan \beta}{\operatorname{sen} \alpha}\right) .
$$

Nas Eqs. (四) e (四), utilizamos as Eqs. (四) e (四).

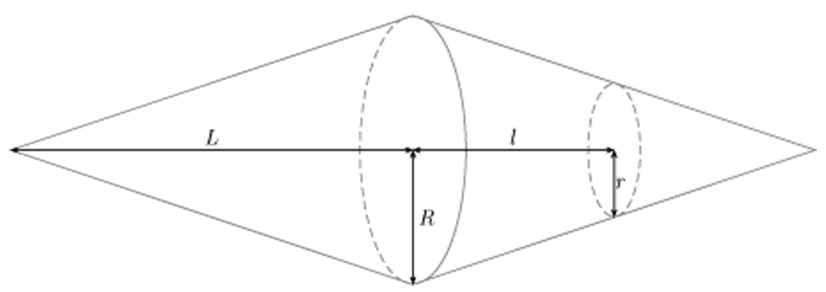

Figura 5 - Vista em perspectiva do duplo cone.

A condição de rolar sem deslizar, implica que a velocidade do duplo cone no ponto de contato é nula. Para que isso aconteça, é necessário que $\mathbf{v}_{c m}+\mathbf{v}_{\text {rot }}=0$, onde $\mathbf{v}_{\text {rot }}=\vec{\omega} \times(-\mathbf{r})$ é a velocidade de rotação do duplo cone no ponto de contato. Como $\mathbf{v}_{c m}=\dot{\mathbf{s}}+\dot{\mathbf{r}}$, temos

$$
\begin{aligned}
\omega r \cos \gamma & =\dot{s}+\dot{r} \operatorname{sen} \gamma \\
-\omega r \operatorname{sen} \gamma & =\dot{r} \cos \gamma .
\end{aligned}
$$

Obtemos as equações acima, projetamos a equação vetorial na direção do vetor $\hat{s}$ e na direção perpendicular ao plano da pista. Resolvendo a Eq. (田), temos

$$
\begin{aligned}
\operatorname{sen} \gamma & =-\frac{\dot{r}}{\dot{s}} \\
\omega & =\frac{\dot{s}}{r} \cos \gamma .
\end{aligned}
$$

E, por fim, usando as Eqs. (四) e (四), obtemos $\gamma$ e $\omega$ em função de $\dot{z}$ e dos parâmetros geométricos do problema

$$
\begin{aligned}
\operatorname{sen} \gamma & =\frac{R}{L} \tan \beta \\
\omega & =\frac{\dot{z}}{R} \frac{\sqrt{L^{2}-R^{2} \tan ^{2} \beta}}{L \operatorname{sen} \alpha-z \tan \beta} .
\end{aligned}
$$

Esses resultados estão de acordo com a Ref. [प], no entanto, nesta referência, partiu-se do princípio de que a superfície do duplo cone e a pista devem ser tangentes no ponto de contato. Como apontado na Ref. [U], em princípo, $-1 \leq \operatorname{sen} \gamma \leq 1$ e $-\infty \leq \tan \beta \leq \infty$, e isso poderia trazer problemas para a primeira linha da Eq. (国). No entanto, para que haja contato entre o duplo cone e a pista, é necessário que $\beta<\arctan (L / R)$
Além disso, estamos admitindo apenas valores positivos para $\beta$. Isso nos leva a $0 \leq \tan \beta<L / R$, e, portanto $0 \leq \operatorname{sen} \gamma<1$, o que não leva a nenhuma inconsistência.

A velocidade do centro de massa em termos de $z, \dot{z}$ e dos parâmetros geométricos é dada por

$$
\begin{aligned}
v_{c m} & =\sqrt{\dot{s}^{2}+2 \dot{s} \dot{r} \operatorname{sen} \gamma+\dot{r}^{2}} \\
& =\frac{\dot{z}}{\operatorname{sen} \alpha} \sqrt{1-\frac{R^{2}}{L^{2}} \tan ^{2} \beta} .
\end{aligned}
$$

Por sua vez, a altura do centro de massa pode ser escrita como

$$
\begin{aligned}
z_{c m} & =z+r \cos (\alpha-\gamma) \\
& =h+\kappa z
\end{aligned}
$$

onde definimos

$$
\begin{aligned}
h & =R\left[\cos \alpha \sqrt{1-\frac{R^{2}}{L^{2}} \tan ^{2} \beta}+\sin \alpha \frac{R}{L} \tan \beta\right] \\
\kappa & \left.=\left[1-\frac{R}{L} \frac{\tan \beta}{\tan \alpha} \sqrt{1-\frac{R^{2}}{L^{2}} \tan ^{2} \beta}-\frac{R^{2}}{L^{2}} \tan ^{2} \beta\right] 0\right)
\end{aligned}
$$

Utilizando as Eqs. (莐), (田), (田) e (四) na Eq. (四) para eliminar $r$ em função de $s$, obtemos a seguinte expressão para energia mecânica

$$
E=\frac{m}{2} \eta^{2} \dot{z}^{2}+V
$$

Na expressão anterior, definimos

$$
V=m g\left(z_{c m}-h\right)=m g \kappa z
$$

$$
\eta=\sqrt{\frac{L^{2}-R^{2} \tan ^{2} \beta}{L^{2} \operatorname{sen}^{2} \alpha}+\frac{R_{g}^{2}}{R^{2}} \frac{L^{2}-R^{2} \tan ^{2} \beta}{(L \operatorname{sen} \alpha-z \tan \beta)^{2}}} .
$$

O momento de inércia de um cone maciço de raio $R$ e massa $m_{c}$ é $I_{c}=3 / 10 m_{c} R^{2}$ [प]]. O duplo cone de massa $m$ pode ser pensado como dois cones de massa $m_{c}=m / 2$ fundidos pela base, portanto, seu momento de inércia é dado por $I=2 I_{c}=6 / 10 m_{c} R^{2}=3 / 10 m R^{2}$. A variável $R_{g}=\sqrt{I / m}$ é chamada de raio de giração, e no caso de um duplo cone maciço, $R_{g}=\sqrt{3 / 10} R$.

\section{Análise do movimento}

Nesta seção, analisaremos a energia mecânica para obtermos algumas informações prévias do movimento do duplo cone. Do comportamento da energia potencial gravitacional (Eq. (एव) ), já é possível perceber a existência de três regimes de movimento distintos. No regime ordinário $(\kappa>0)$, a energia potencial aumenta linearmente $\operatorname{com} z$. No regime indiferente $(\kappa=0)$, a energia potencial gravitacional é nula. No regime paradoxal $(\kappa<0)$, a energia potencial diminui linearmente $\operatorname{com} z$.

Existe uma limitação geométrica relacionada a região onde o duplo cone pode manter o contato com 
a pista. Não é difícil de verificar das Figs. 2 e 3 que isso ocorre desde que $0 \leq z \leq L \operatorname{sen} \alpha / \tan \beta$. A energia mecânica do duplo cone também impõe restrições à região espacial onde ele pode se mover. Observe que as energias cinéticas de translação e de rotação são sempre positivas [प], portanto, qualquer movimento deve ocorrer dentro de regiões onde $E \geq V$. Chamamos de pontos de retorno $z_{r}$, os pontos em $E=V$, ou seja, os pontos onde a energia cinética, e consequentemente a velocidade, são nulas. Da Eq. (प्⿱一𫝀), temos

$$
z_{r}=\frac{E}{m g \kappa}
$$

Usando a Eq. (ए2), é possível verificar que no regime ordinário $(\kappa>0)$ com $0 \leq E<m g \kappa L \operatorname{sen} \alpha / \tan \beta$, o duplo cone pode mover-se no intervalo $0 \leq z \leq z_{r}$. Já no regime paradoxal $(\kappa<0) \operatorname{com} m g \kappa L \operatorname{sen} \alpha / \tan \beta \leq E<$ 0 , o movimento é possível apenas dentro do intervalo $z_{r} \leq z \leq L \operatorname{sen} \alpha / \tan \beta$. Para qualquer outra situação, ou seja, regime ordinário $\operatorname{com} E \geq m g \kappa L \operatorname{sen} \alpha / \tan \beta$, regime paradoxal com $E \geq 0$, ou regime indiferente $(\kappa=0)$ com $E \geq 0$, o duplo cone pode mover-se por toda a extensão útil da pista $(0 \leq z \leq L \operatorname{sen} \alpha / \tan \beta)$.

\section{Descrição do movimento}

Nesta seção, utilizaremos a conservação da energia mecânica para estudar o movimento do duplo cone. Mais especificamente, estudaremos a evolução temporal da altura de contato, da altura do centro de massa, da velocidade do centro de massa e da velocidade angular de rotação do duplo cone.

Usaremos como condições iniciais $z(0)=z_{0}$ e $\dot{z}(0)=v_{0}$, e resolveremos numericamente as equações diferencial

$$
\eta^{2}\left(z_{0}\right) v_{0}^{2}=\eta^{2}(z) \dot{z}^{2}+2 g \kappa\left(z-z_{0}\right) .
$$

A partir da solução de $z$, é possível calcular $\omega, v_{c m}$ e $z_{c m}$ utilizando as Eqs. (四), (四) e (四). A seguir, apresentaremos separadamente os resultados obtidos para os três regimes de movimento do duplo cone.

\subsection{Regime ordinário $(\kappa>0)$}

Na Fig. 6, apresentamos os resultados numéricos das alturas do ponto de contato e do centro de massa do duplo cone em função do tempo. Na Fig. 7, apresentamos a dependência temporal da velocidade do centro de massa e da velocidade angular.

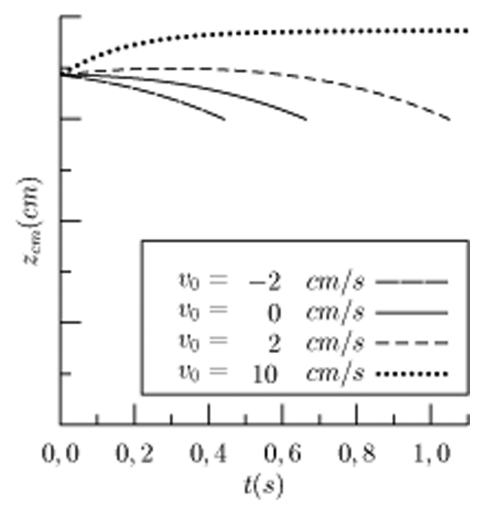

Figura 6 - Gráfico de $z$ e $z_{\mathrm{cm}}$ em função do tempo. Neste gráfico usamos $R=3 \mathrm{~cm}, L=4 \mathrm{~cm}, \alpha=15^{\circ}$ e $\beta=15^{\circ}$.
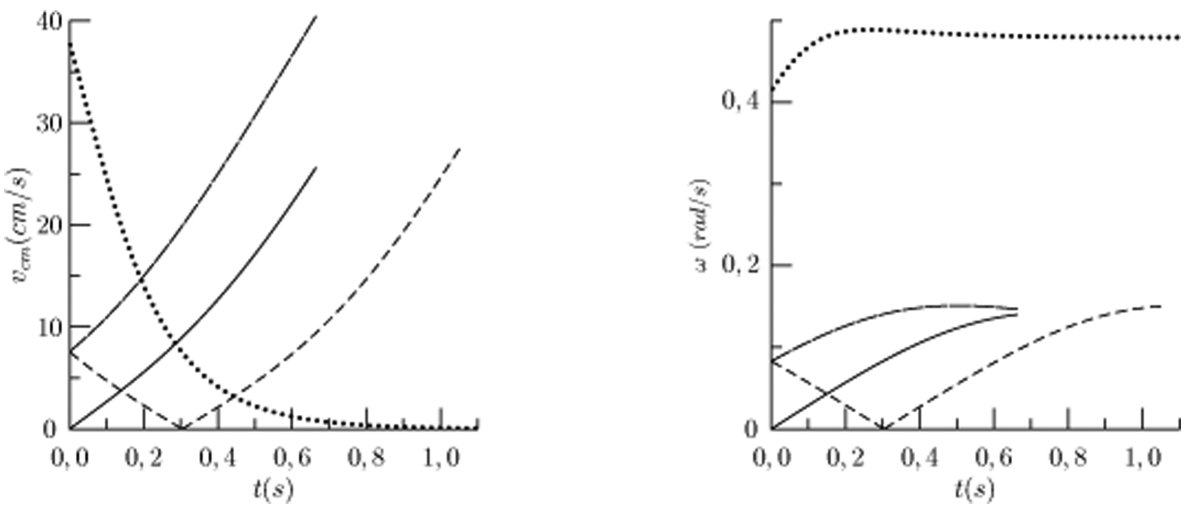

Figura 7 - Gráfico de $v_{\mathrm{cm}}$ e $\omega$ em função do tempo. Neste gráfico usamos $R=3 \mathrm{~cm}, L=4 \mathrm{~cm}, \alpha=15^{\circ}$ e $\beta=15^{\circ}$. 
Como podemos ver, para as velocidades iniciais $v_{0}=-2 \mathrm{~cm} / \mathrm{s}$ e $v_{0}=0 \mathrm{~cm} / \mathrm{s}$, o duplo cone parte de $z_{0}$ e desce até chegar no ponto mais baixo da rampa. Nos dois casos $v_{\mathrm{cm}}$ sempre aumenta, mas comparando os dois casos, percebemos que com $v_{0}=-2 \mathrm{~cm} / \mathrm{s}, v_{\mathrm{cm}}$ é sempre maior e o duplo cone faz o mesmo percurso em um tempo menor. Para $v_{0}=2 \mathrm{~cm} / \mathrm{s}$, inicialmente, o duplo cone sobe perdendo velocidade até chegar ao ponto de retorno. Depois disso, o mesmo passa a descer a rampa ganhando cada vez mais velocidade. Por fim, para $v_{0}=10 \mathrm{~cm} / \mathrm{s}$, o duplo cone sobe a rampa, mas não torna a desce-la. Neste último caso, sua velocidade sempre diminui indo assintoticamente para zero e, teoricamente, o duplo cone nunca chega ao ponto mais alto da rampa. É possível perceber ainda que a altura do centro de massa do duplo cone segue um comportamento muito parecido com a altura do ponto de contato, no entanto, $z_{\mathrm{cm}}>z$. Em relação a velocidade angular, podemos perceber nos 4 casos apresentados, que ela diminui enquanto o duplo cone sobe a pista e aumenta quando ele desce.

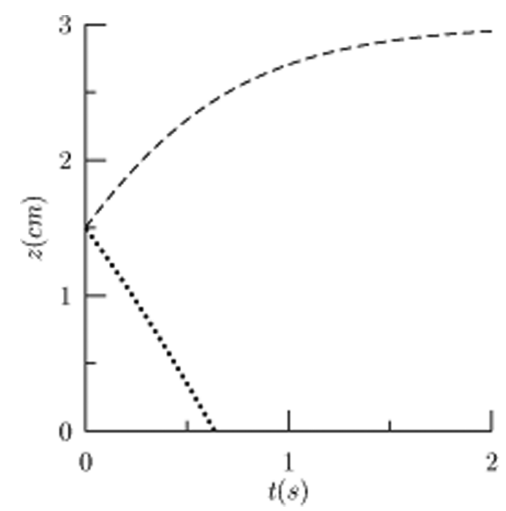

\subsection{Regime Indiferente $(\kappa=0)$}

Nas Figs. 8 e 9, apresentamos os resultados numéricos de $z(t), z_{\mathrm{cm}}(t), v_{\mathrm{cm}}(t)$ e $\omega(t)$ para o regime indiferente.

Como podemos ver, para $v_{0}=-2 \mathrm{~cm} / \mathrm{s}$, o duplo cone parte do ponto inicial $z_{0}$ e desce até chegar ao vértice da pista, e velocidade do centro de massa do duplo cone, $v_{\mathrm{cm}}$ sempre aumenta. Já para $v_{0}=2 \mathrm{~cm} / \mathrm{s}$, o movimento ocorre no sentido do ponto inicial $z_{0}$ ao ponto mais alto da pista. Como no regime ordinário, $v_{\text {cm }}$ diminui sempre diminui e tende assintoticamente a zero, sendo assim, o duplo cone nunca chega ao final da rampa. No que diz respeito a altura do centro de massa, podemos verificar que independente do movimento do duplo cone, ela permanece constante. Com relação à velocidade angular do duplo cone, percebemos que ela aumenta quando o duplo cone sobe a pista e diminui quando ele desce, ao contrário do que ocorre no regime ordinário.

\subsection{Regime paradoxal $(\kappa<0)$}

Nas Figs. 10 e 11, apresentamos os gráficos de $z(t)$, $z_{\mathrm{cm}}(t), v_{\mathrm{cm}}(t)$ e $\omega(t)$ para o regime paradoxal.

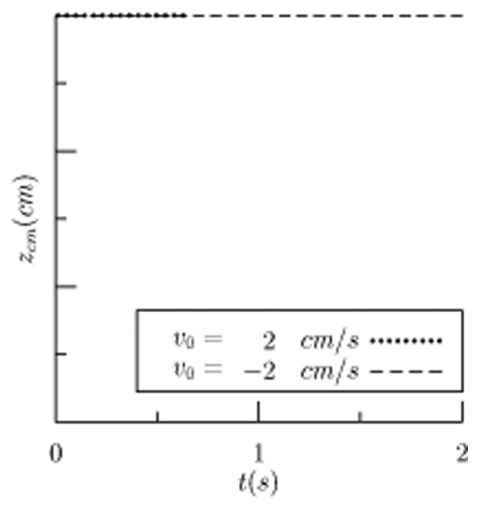

Figura 8 - Gráfico de $z$ e $z_{c m}$ em função do tempo. Neste gráfico usamos $R=3 \mathrm{~cm}, L=4 \mathrm{~cm}, \alpha=\arcsin \left(0,75 \tan \left(15^{\circ}\right)\right)$ e $\beta=15^{\circ}$.
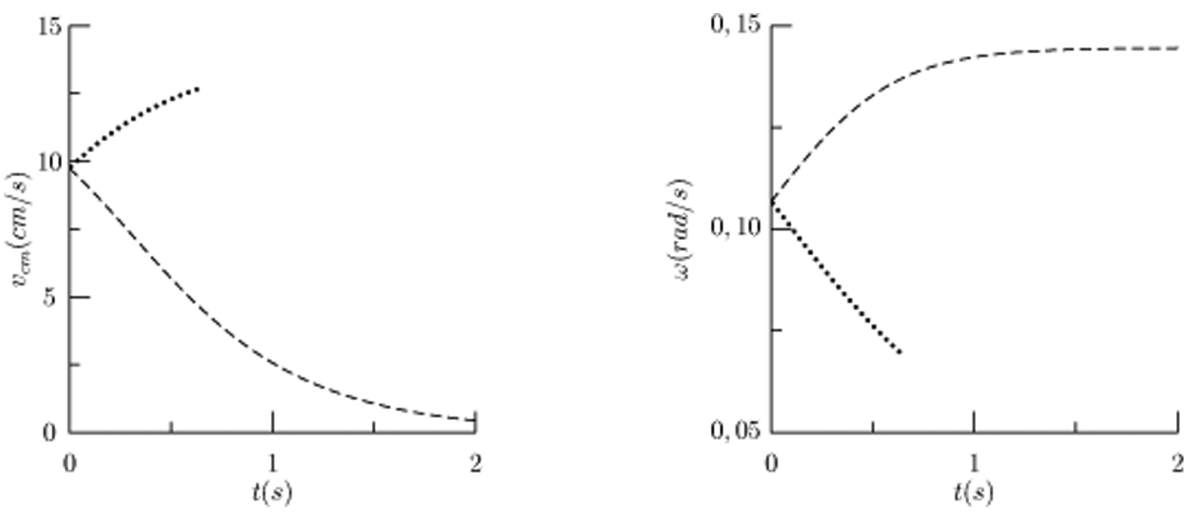

Figura 9 - Gráfico de $v_{\mathrm{cm}}$ e $\omega$ em função do tempo. Neste gráfico usamos $R=3 \mathrm{~cm}, L=4 \mathrm{~cm}, \alpha=\arcsin \left(0,75 \tan \left(15^{\circ}\right)\right)$ e $\beta=15^{\circ}$. 

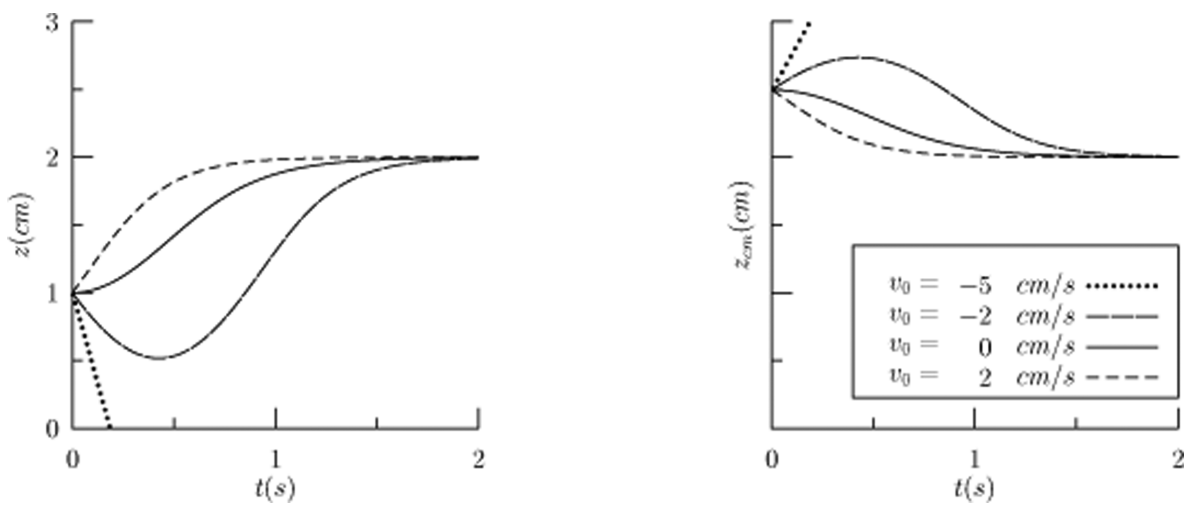

Figura 10 - Gráfico de $z$ e $z_{\mathrm{cm}}$ em função do tempo. Neste gráfico usamos $R=3 \mathrm{~cm}, L=4 \mathrm{~cm}, \alpha=7^{\circ} 42^{\prime}$ e $\beta=15^{\circ}$.
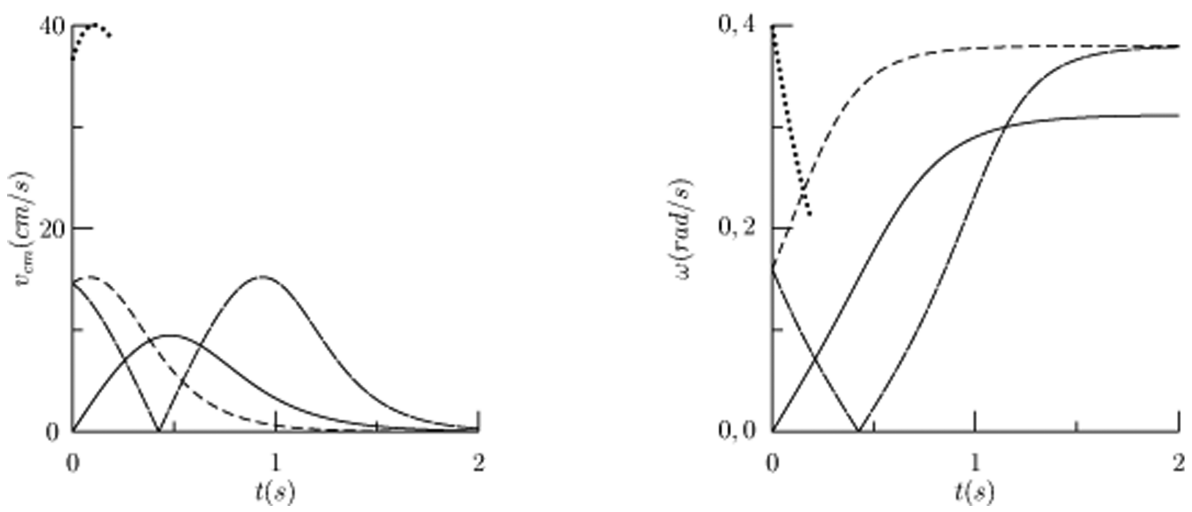

Figura 11 - Gráfico de $v_{\mathrm{cm}}$ e $\omega$ em função do tempo. Neste gráfico usamos $R=3 \mathrm{~cm}, L=4 \mathrm{~cm}, \alpha=7^{\circ} 42^{\prime}$ e $\beta=15^{\circ}$.

Como podemos ver das Figs. 10 e 11, para a velocidade inicial de $v_{0}=-5 \mathrm{~cm} / \mathrm{s}$, o duplo cone dispõe de energia suficiente para descer do ponto inicial até o vértice da rampa. Para $v_{0}=-2 \mathrm{~cm} / \mathrm{s}$, o duplo cone parte do ponto inicial, desce até o ponto de retorno e depois sobe a rampa. Para $v_{0}=0 \mathrm{~cm} / \mathrm{s}$ e $v_{0}=2 \mathrm{~cm} / \mathrm{s}$, o duplo cone simplesmente sobe a rampa. Em relação a altura do centro de massa, percebemos que assim como no regime ordinário, $z_{\mathrm{cm}}>z_{c}$, no entanto, as velocidades $\dot{z}$ e $\dot{z}_{c m}$ aparecem espelhadas: se o duplo cone sobe a rampa, seu centro de massa desce, e vice versa. Em relação a velocidade do centro de massa percebemos que assim como nos dois outros regimes, $v_{\mathrm{cm}}$ tende a zero conforme o duplo cone se aproxima do ponto mais elevado da pista. Na Ref. [8], os autores também preveem este comportamento (para $v_{0}=0 \mathrm{~cm} / \mathrm{s}$ ) e explicam que na realidade o duplo cone para um pouco antes. Isso ocorre porque não levamos em consideração nos cálculos forças dissipativas como resistência do ar. Assim como ocorre no regime indiferente, a velocidade angular de rotação do duplo cone aumenta quando o duplo cone sobe a pista e diminui quando ele desce.

\section{Verificações experimentais}

Montamos um experimento do duplo cone com a finalidade de confirmar experimentalmente parte de nossos resultados teóricos. Utilizamos um duplo cone de um brinquedo no estilo kit de ciências para crianças, que é similar a dois funis juntos pela base, e com ângulo de abertura equivalente ao de um duplo cone ideal com $R=3 \mathrm{~cm}$ e $L=4 \mathrm{~cm}$. O problema maior foi a pista em $\mathrm{V}$, pois a do kit era composta de duas varetas de plástico, curtas e flexíveis. A solução que encontramos foi utilizar outro brinquedo para construir a rampa: blocos de montar (Fig. 12).

Em um primeiro momento, utilizamos o aparato experimental para verificar o resultado expresso pela Eq. (四), ou seja, as configurações geométricas para as quais o paradoxo gravitacional ocorre ou não. A partir da abertura da rampa foi possível controlar o ângulo de abertura $\beta$ e adicionado blocos a base foi possível controlar o ângulo de ascensão da pista $\alpha$. Ao variarmos as configurações geométricas, verificávamos se o duplo cone tendia a subir ou descer a rampa em V. Os resultados teóricos e experimentais estão representados na Fig. 13.

Como podemos ver da Fig. 13, os resultados ex- 
perimentais estão de acordo com nossa teoria. Além disso, nosso resultado estão de acordo com os resultados apresentados nas Refs. [0] e [四]. Curiosamente, na Ref. [四], os autores cometem um erro conceitual, equivalente a assumir que $\gamma=\alpha$. Isso implicaria $\kappa=1-(R / L)(\tan \beta / \operatorname{sen} \alpha)$, o que levaria a erros no estudo do movimento do duplo cone, mas leva ao resultado correto se o que está em estudo for o sinal de $\kappa$.

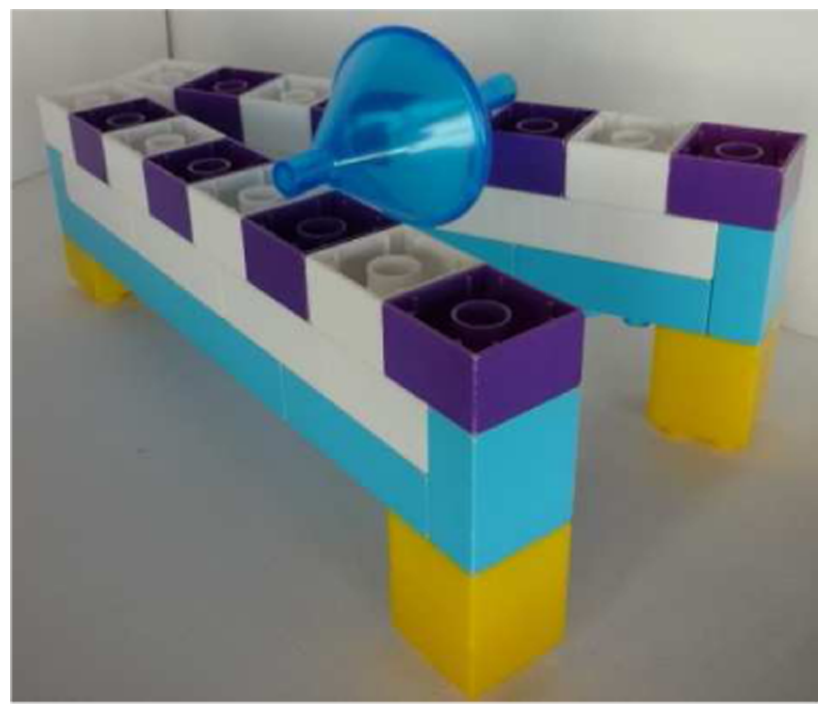

Figura 12 - Aparato experimental constituído basicamente de brinquedos.

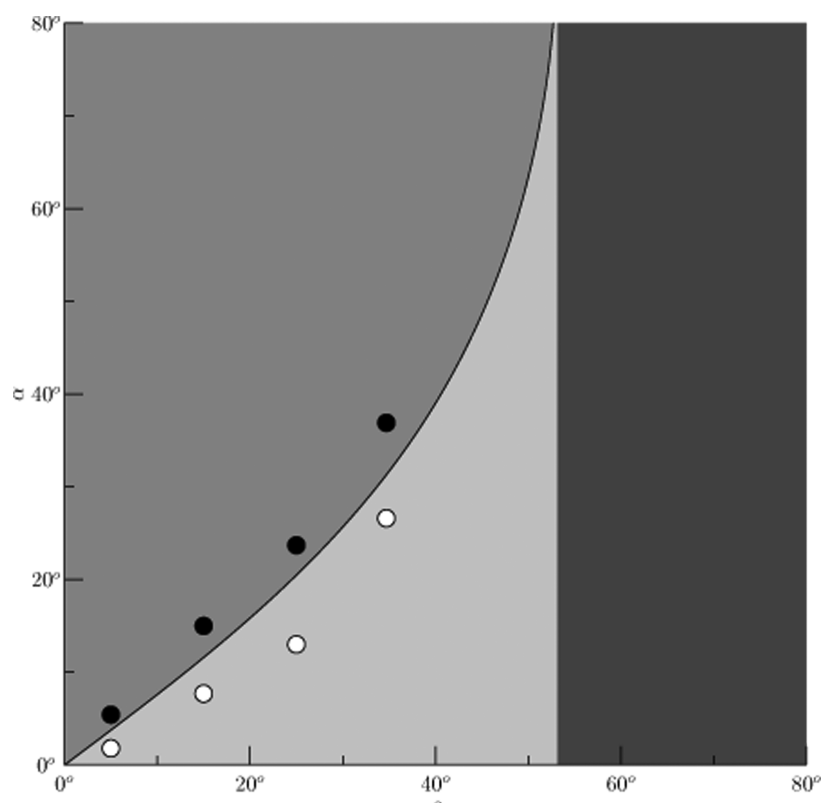

Figura 13 - Diagrama dos diferentes regimes de movimento do duplo cone para $R / L=3 / 4$. Da mais clara para a mais escura, as áreas do diagrama representam a região onde ocorre o regime paradoxal, o regime ordinário e a área onde não há contato entre o duplo cone e a pista. Os pontos representam os dados experimentais: Os brancos indicam a tendência do duplo cone de subir a rampa em $\mathrm{V}$ e os pretos indicam a tendência a descer.
Em um segundo momento, utilizamos o aparato experimental para confirmar qualitativamente boa parte dos resultados referentes ao movimento do duplo cone. Conseguimos observar tudo o que foi relatado para o regime ordinário. No regime paradoxal, só não conseguimos observar o movimento próximo ao final da extensão útil da pista, uma vez que o duplo cone utilizado não tinha o formato ideal. Nada pudemos verificar sobre o regime indiferente, pois o mesmo ocorre para uma condição muito específica, difícil de ser ajustada.

\section{Conclusões}

Neste artigo, utilizamos o princípio da conservação de energia para estudar o movimento de um duplo cone sobre uma rampa inclinada em formato de V. Como pudemos ver, no regime em que $\kappa>0$, o movimento do duplo cone não difere muito daquele de um corpo sobre um plano inclinado: se ele parte do repouso ou é empurrado para baixo, o mesmo descerá até a parte mais baixa da rampa. No caso de um impulso não muito intenso para cima, o mesmo sobe até um determinado ponto da rampa e depois desce até a parte mais baixa. No caso de um impulso suficientemente forte, o mesmo sobe toda a extensão da rampa. Dados os motivos expostos acima, batizamos esse regime de regime ordinário.

No regime em que $\kappa=0$, o duplo cone apresenta não apresenta nenhuma tendência natural de subir ou descer a rampa. Na verdade, neste regime a altura do centro de massa do duplo cone e, portanto sua energia potencial gravitacional, permanecem constantes. Se o soltarmos do repouso, o mesmo ficará parado indefinidamente. No caso do duplo cone receber um impulso, ele seguirá até o final do percurso naquela mesma direção. Por esses motivos, chamamos esse regime de regime indiferente.

Os resultados mais interessantes ocorrem no regime paradoxal $(\kappa<0)$. Se soltarmos o duplo cone do repouso, ele se moverá em direção a porção mais alta da rampa. Um efeito ainda mais surpreendente ocorre quando damos ao duplo cone um impulso moderado para baixo, ele começa a mover-se para baixo, mas em algum momento passa a mover-se para o alta do rampa. O paradoxo gravitacional é desvendado ao considerarmos a posição do centro de massa do duplo cone: devido às configurações geométricas, a medida que o duplo cone sobe a rampa, seu centro de massa na verdade desce.

\section{Referências}

[1] A. Medeiros e C.F. Medeiros, Revista Brasileira de Ensino de Física 25, 333 (2003).

[2] J.A. Desaguliers, Course of Experimental Philosophy, Tomo I (W. Innys, Londres, 1734). 
[3] William Leybourn e Richard Sault, Pleasure with Profit: Consisting of Recreations of divers kinds, viz. (R. Baldwin and J. Dunton, Londres, 1694), Tomo III, p. 12, disponível em https://archive.org/details/ pleasurew1thpro00saulgoog, acessado em 10/4/2014.

[4] J. Canalle e R. Moura, Caderno Catarinense de Ensino de Física 15, 323 (1998).

[5] E.C. Valadares, Física Mais que Divertida: Inventos Eletrizantes Baseados em Materiais Reciclados e de Baixo Custo (Editora UFMG, Belo Horizonte, 2002).

[6] Giuseppe Pino, L'incredibile Doppio Cono AntiGravitazionale. Publicação on-line no site Paper Pino, disponível em paperpino.net, acessado em 10/1/2014.
[7] Sohang C. Gandhi e Costas J. Efthimiou, Europian Journal of Physics 26, 681 (2004).

[8] J. Bartos e J. Musilova, Europian Journal of Physics 25, 675 (2004).

[9] H.M. Nussenzveig, Curso de Física Básica - Mecânica (Ed. Edgard Blucher, São Paulo, 2002), v. 1, $4^{\mathrm{a}}$ ed.

[10] A. Chaves e J.F. Sampaio, Física Básica Mecânica (LTC, Rio de Janeiro, 2004), v. 1, $1^{\mathrm{a}}$ ed.

[11] J.B. Marion e S.T. Thornton, Dinâmica Clássica de Partículas e Sistemas (Cengage Learning, São Paulo, 2011), $1^{\text {a }}$ ed. 